\title{
The Use of Orthogonal Decomposition Pattern Short Wave for Sumba's Fabric
}

\author{
Yustina Rada, Harrizki Arie Pradana
}

\begin{abstract}
Ikat Sumba weaving has a diversity of style that is unique and appealing. Patterns or designs woven cloth image depicts symbols of ancestral cultural attributes of the people of Sumba with their respective meanings that cannot be found in other countries. But in its use, not many people especially young people and tourists who know how the origin of the fabric Sumba. Moreover, to know more about the various types of motives and philosophy. From this background, the author would like to develop an Application Recognition Motif Cloth Sumba by applying the method based on similar texture image retrieval using wavelet. Kind used wavelet is Haar wavelet decomposition to function. This application is expected to provide specialized services and information to the public both local and foreign people who want to get information about local woven fabrics Sumba.
\end{abstract}

Keywords-Haar wavelet, texture, Sumba's woven fabric pattern.

\section{INTRODUCTION}

Imagery database is currently very widely used, from time to time its use continues to grow with more and more people are utilizing their usefulness. Its use varies depending on the application. Along with the continued development of the size of the base image data, the traditional methods commonly used in image retrieval is no longer possible to use, for example image search by keyword, sometimes the keyword (text) which we entered does not fit the image we expect that with provide keywords alone are not enough, should be developed with other methods for retrieving images that can be used as a substitute or added to the keyword system. For that developed a new method that Haar wavelet [1].

Sumba is one of the islands located in eastern Indonesia, precisely in the province of East Nusa Tenggara (NTT). The beauty of Sumba Island itself is already well known to every corner of the world, is evidenced by the many foreign tourists who come to enjoy the exotic island of Sumba [2].

Sumba Island has a lot of diversity of culture and tourism, one culture is no less interesting is the area Sumba woven fabric itself. Said to be interesting, because at every motif woven area will describe the culture at one of the area corresponding to the origin of manufacture of woven fabrics itself.

Yustina Rada, Wira Wacana University, Waingapu, East Sumba, NTT Indonesia, Email: yustinarada@gmail.com

Harrizki Arie Pradanam, STMIK Atma Luhur, Pangkalpinang, Bangka, Bangka Belitung, Indonesia, Email: harrizkiariep@atmaluhur.ac.id
Lack of information created in a variety of media to make the public cannot get information about the area Sumba woven fabric needs to be made clear that the alternative media to inform the promotion of regional Sumba woven fabric, so that the public and tourists can find local motifs woven fabric Sumba.

The formulation of the issues contained in this research is to find the image of homogeneous texture in the image and also to enter the library of image (base image data). Then, the use of wavelet decomposition function to get the value of wavelet coefficients, and the last step to get calculating the value of the distance between the image obtained from the value of the average (mean) and standard deviation of the results of wavelet decomposition functions. Boundary problem for the present study were, the image of the database used in the form of texture image and the non-textured with JPG or JPEG format that is in the current directory, then structural color for texture images are Red, Green, Blue (RGB) and homogeneous. The number of image texture that is used by 2 pieces of imagery, with the level of similarity that is different. The last is the number of results displayed image as much as 18 pieces of the image with the highest similarity value.

The purpose of this study was to test the effectiveness of using wavelet decomposition function in the process of matching the image against a database of images, especially the texture image (image with homogeneous texture). Getting the image of a texture similar to the texture image query with the same degree of truth with a similar image by the human eye sight and the last is to build an application program on a texture image by using wavelet decomposition functions.

\section{LITERATURE REVIEW}

The discovery occurred in early 1998 when Daubenchies create orthonormal wavelet family and compact support, the result is an inspiration from Meyer and Mallat [3]. A wave or wave is usually defined as an oscillation function of time (space) with the existing system. The system is defined as a unit consisting of one or two components or sub-systems that interact to achieve a goal. Of the many studies, could mean that the Haar wavelet transformation, there are two processes that should be carried forward transformation (decomposition) and inverse transformation (reconstruction) [4]. Forward transformation is useful to break the image. While the inverse transformation is the opposite, namely to reshape the image fragments from the process forward into a normal image (reconstruction) [5].

The division of the signal into high-frequency and low frequency in the filtering process high-pass filter and low-pass 
filter called decomposition. The decomposition process starts by passing the signal from passing through high-pass filter and low-pass filter [6]. Each step in the transformation into account collection Haar wavelet coefficients and the set average. If a data set $\mathrm{s}_{-} 0, \mathbb{}$, $\mathrm{s}_{-} 1, \ldots, \mathrm{s}_{-}(\mathrm{N}-1)$ contain elements $\mathrm{N}$, there will be $\mathrm{N} / 2$ average and $\mathrm{N} / 2$ values of coefficients. The average is stored in the lower half of the unity of $\mathrm{N}$ and the coefficients are stored in the half on top.

The average becomes the input for the next step in the calculation of wavelet, which for iteration $\mathrm{i}+1, \mathrm{~N}_{-}(\mathrm{i}+1)=\mathrm{N}$ i / 2. Iteration-iteration continues until an average of single and single coefficients are calculated. It will replace a set of data origin of the elements of $\mathrm{N}$ to the average obtained, followed by a set of coefficients which measure is the increase in power of two (e.g., $2 \wedge 0, \llbracket 2 \rrbracket \wedge 1, \llbracket 2 \rrbracket \wedge 2, \ldots, N / 2$ ) [7].

\section{A. Texture}

According to [8], the texture analysis performs an important place in many tasks such as machine vision, scene classification, shape determination and image processing. The major application domains in texture analysis are Texture classification, Texture segmentation, Shape recovery. The problem in texture classification is that textures in real world are often not uniform due to variation in orientation, scale and other visual appearance [8].

\section{B. PSNR (Peak Signal to Noise Ratio)}

According to [9], PSNR is one of the parameters that can be used to quantify image quality. PSNR parameter is often used as a benchmark level of similarity between reconstructed image and the original image then the larger PSNR will produce better image quality.

\section{Wavelet}

Wavelet is a mathematical tool which is able to perform a function in the decomposition of the hierarchy. Wavelet can be used to describe a model or the original image into a mathematical function regardless of the shape of the model is an image, a curve, or a field [10]. The wavelet transform is a conversion function that can split function or signal in the frequency component or a different scale, and can further be studied each of the components with a specific resolution in accordance with the scale. Wavelet has many types depending on the function that uses as Haar wavelet, Wavelet Symlet, Deubechies Wavelet, Wavelet Coifflet, and so forth. In this research method used is the Haar wavelet.

\section{D.Haar Wavelet Transformation}

Wavelet transform data from spatial domain to the frequency domain and then store each component in accordance with the scale resolution. Wavelet has orthogonal basis in certain vector space. The simplest wavelet transform is the Haar wavelet [11]. General functions of the Haar wavelet can be seen in the equation 1.

$$
\begin{gathered}
\left\{\Psi_{k+1}(l)\right\}_{l=0}^{2^{k+1}-1} \\
\left\{\Psi_{k}(l)\right\}_{l=0}^{2^{k}-1} \quad \begin{array}{c}
\text { where, } \\
\text { and }
\end{array}
\end{gathered}
$$

$$
\left\{W_{k, l}\right\}_{l=0}^{2^{k}-1} \quad \text { detail signal }
$$

As for the operator approximation (averaging) and operator details, as follows:

$$
\begin{aligned}
& \Psi_{k}(l)=\frac{\Psi_{k+1}(2 l)+\Psi_{k+1}(2 l+1)}{2}, 0 \leq l \leq 2^{k}-1 \\
& W_{k, l}=\Psi_{k+1}(2 l)-\Psi_{k+1}(2 l+1), 0 \leq l \leq 2^{k}-1
\end{aligned}
$$

\section{E. Haar Wavelet Transformation for Image Decomposition}

In image processing, correlation signal into sub-sampled signal is also called the decomposition process. Signals process decomposition with the Haar wavelet method can be used in the transformation of the image by applying wavelet decomposition in 2D. 2D wavelet decomposition process established through 1D Haar wavelet transformation.

The general shape of 1D Haar wavelet transformation is shown in Figure 1. The wavelet transform 1D can be extended to form a $2 \mathrm{D}$ wavelet transform using wavelet filters were separated. 2D wavelet can be calculated by applying a 1D transformation to all lines of the input image (the image length or dimension $\mathrm{x}$ ), and then repeat it on all columns (the width of the image or dimensions) [12].

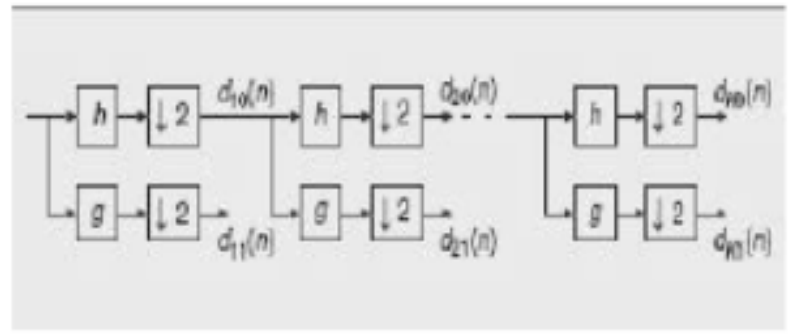

Fig. 1 Haar Wavelet 1D Decomposition

As an example, in Figure 2, which shows the first level ( $\mathrm{K}=$ 1) of the wavelet transform $2 \mathrm{D}$, with a corresponding notation in the image 4.

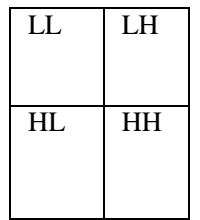

LL : low-pass result of the row and column

LH : the results forwarded to the low-pass to high-pass line against column

$\mathrm{HL}$ : high-pass result of the line followed by a low-pass against column

$\mathrm{HH}$ : high-pass result of the row and column

Fig. 2 Wavelet Decomposition at 2 Dimension on level 1

To make the process of Haar wavelet decomposition, then used the averaging method (average) and differencing (difference or difference) for each row and column of the image. Mathematically we can formulate a method of averaging and differencing.

\section{F. RGB Color Model}

In the RGB model, each color shows the spectral components of the primary red, green and blue. This model is based on a Cartesian coordinate system. The image that is represented in the RGB color model consists of three components of the image, respectively for each primary color $(\mathrm{R}, \mathrm{G}, \mathrm{B})$. When displayed on a monitor RGB, three combinations of this image are in the phosphor screen to 
produce a color composite image. The number of bits used to represent each pixel in RGB space called pixel depth. Note that the RGB images where each image of red, green, blue is the 8-bit image. Under conditions of each color pixel RGB then, triplet of values (RGB) has a depth of 24-bit (3-layer image with the number of bits per layer). A full-color image is often used to express the image of 24-bit RGB color. The total number of colors in a 24-bit image is $\left(2^{\wedge} 8\right)^{\wedge} 3=16,777,216$ [13].

\section{G.Motive and Meaning of Sumba's Fabric}

On Sumba cloth, there are a variety of motives and meanings contained therein. For more details in about a variety of motifs Sumba, can be seen in Table 1 .

Table 1. Motive and Meaning fabrics Sumba Ikat Weaving

\begin{tabular}{|c|c|c|}
\hline No & Nama Kain & Keterangan \\
\hline 1. & Hinggi Kombu & $\begin{array}{l}\text { Dye: Kombu or Noni roots } \\
\text { Used: as everyday clothing, } \\
\text { clothing visit or visits, party wear } \\
\text { traditional marriage or death, is } \\
\text { now also used as souvenirs for the } \\
\text { guests, acquaintances, or friends. } \\
\text { Wear during ritual "marapu". } \\
\text { Element motifs: horses, chickens. } \\
\text { Philosophical meaning: shades } \\
\text { horse is a symbol of virility, } \\
\text { courage, prosperity and agility; } \\
\text { chicken complexion symbolizes } \\
\text { awareness, virility, a sign of life, it } \\
\text { is expected the wearer look good } \\
\text { and be respected. }\end{array}$ \\
\hline 2. & Hinggi Kawuru & $\begin{array}{l}\text { Dyes: indigo or indigo. } \\
\text { Used: as everyday clothing, } \\
\text { clothing visit or visits, party wear } \\
\text { traditional marriage or death, is } \\
\text { now also used as souvenirs for the } \\
\text { guests, acquaintances, or friends. } \\
\text { Wear during ritual "marapu". } \\
\text { Motif elements: chicken, horses. } \\
\text { Philosophical meaning: horse } \\
\text { shades are placed on the cloth } \\
\text { background blue color is a symbol } \\
\text { of grandeur, greatness, pride, and } \\
\text { social status. }\end{array}$ \\
\hline 3. & & $\begin{array}{l}\text { Dye: kombu or noni roots. } \\
\text { Use: as a body, had been wrapped } \\
\text { in a sacred ritual. } \\
\text { Philosophical meaning: magic, } \\
\text { where the human figure in its } \\
\text { clawed position waist is a symbol } \\
\text { of the clan who has the task to } \\
\text { resolve the rooftops. }\end{array}$ \\
\hline 4. & $\begin{array}{l}\text { Kombu fabric Shrimps } \\
\text { pattern } \\
\text { (3) }\end{array}$ & $\begin{array}{l}\text { Dye: kombu or noni roots. } \\
\text { Used: as everyday clothing, } \\
\text { clothing visit or visits, party wear } \\
\text { traditional marriage or death, is } \\
\text { now also used as souvenirs for the } \\
\text { guests, acquaintances, or friends. } \\
\text { Wear during ritual "marapu". } \\
\text { Motif elements: shrimp. } \\
\text { Philosophical meaning: the style } \\
\text { shrimp is a symbol of the attitude } \\
\text { of a leader who behaves mature or } \\
\text { adult. }\end{array}$ \\
\hline
\end{tabular}

\section{RESEARCH METHODOLOGY}

The process flow system was first conducted by entering data in the form of image on Sumba's fabric cloth, namely Hinggi Kombu. Once the data is contained in a single folder cloth, the Haar wavelet decomposition process is done by using MATLAB. The results of the decomposition are then forwarded for part of the image to get approximation with its output forwarded by finding the mean and standard deviation, so the output is issued or produced was almost the same as the results of the data fabric first entered due to the higher value MSE greater the difference between the two images being compared. For more details, the overall process flow system in Figure 3.

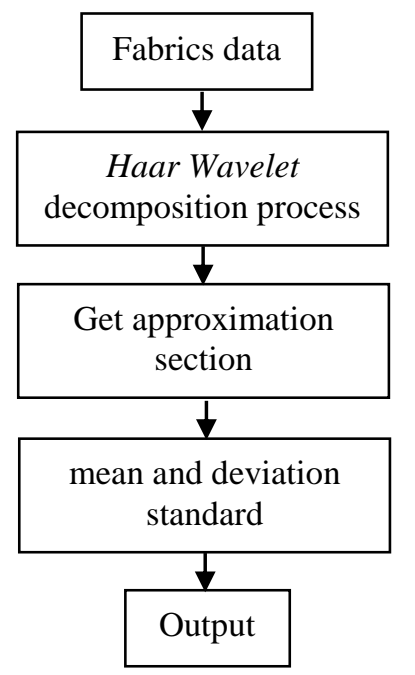

Fig. 3 Overall process flow system

The method used in the making of this study are:

1. Library Research Methods

This method is used to search the literature or literature sources related to and reinforce the existing theories and obtain real data.

2. Data Collection Techniques

The method used for the process of collecting data relevant to the process of triangulation, such as interviews, observation or observation, and documentation.

3. Data Analysis

Analysis of the data in this study using quantitative methods conducted jointly with the way the data collection process to the stage of data collection, data reduction, data presentation, and decision-making or verification using MATLAB software version R2012b. 


\section{RESULTS}

Analysis of the old system work process flow for tourists or visitors who want to know the places producing woven fabric Sumba area:

1. The tourists or visitors coming to the island of Sumba who wish to buy or know the places manufacture of woven fabrics area by visiting the Office of Tourism East Nusa Tenggara province or hotels and inns that provide information about tourism and tourist spots producing woven fabric area.

2. The tourists can directly ask the locals about the places manufacture of woven fabrics area that they need.

Here is the algorithm of the program:

1. Read the variations of wavelet image;

2. The image is read and variations of wavelet-specified, then enter the picture;

3. Enter the level of decomposition;

4. Enter wavelet variation;

5. Decomposition calculated by MSE and PSNR parameter; and

6. Show results enter and plot.

Following the process by programming algorithm, it can be seen the results as shown in Figure 4.

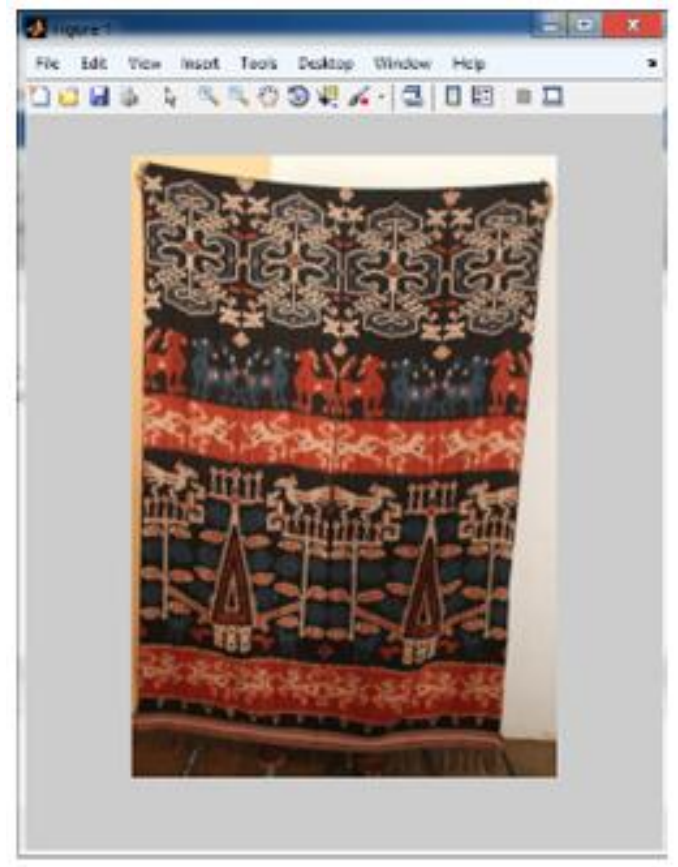

Fig. 4 Results Data Output be in the form of Image

Afterwards, see a comparison of the RGB colors that occur after the calculations performed MATLAB. The result can be seen in Figure 5.

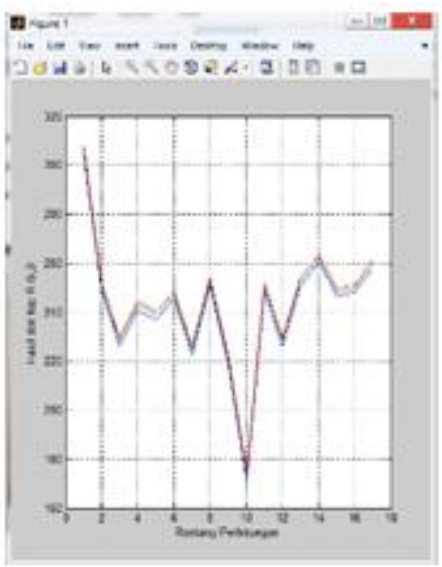

Fig. 5 RGB Color Comparison

After seeing the results of the comparison of RGB color, then the next is following the data presenting from RGB to the total and mean. These results can be seen in Figure 6. In Figure 6, it is clearly written comparison between the total and the mean on each color, either red, green, and blue. The results of the three colors are not too far away.

\begin{tabular}{|r|r|r|c|c|c|}
\hline 1 & Red & Green & Blue & total & mean \\
\hline 2 & 304.0769 & 306.3561 & 307.8214 & 918.2544 & 306.0848 \\
\hline 3 & 248.4026 & 250.3293 & 251.7834 & 750.5153 & 250.1718 \\
\hline 4 & 226.0983 & 228.2317 & 229.7723 & 684.1023 & 228.0341 \\
\hline 5 & 240.6445 & 242.7778 & 244.3498 & 727.7721 & 242.5907 \\
\hline 6 & 236.9181 & 239.1004 & 240.6765 & 716.695 & 238.8983 \\
\hline 7 & 245.379 & 247.2161 & 248.6277 & 741.2228 & 247.0743 \\
\hline 8 & 222.4987 & 224.516 & 226.0406 & 673.0553 & 224.3518 \\
\hline 9 & 250.8519 & 252.8004 & 254.2334 & 757.8857 & 252.6286 \\
\hline 10 & 218.068 & 220.0013 & 221.4539 & 659.5232 & 219.8411 \\
\hline 11 & 171.1623 & 173.1393 & 174.6945 & 518.9961 & 172.9987 \\
\hline 12 & 248.4026 & 250.3293 & 251.7834 & 750.5153 & 250.1718 \\
\hline 13 & 226.0983 & 228.2317 & 229.7723 & 684.1023 & 228.0341 \\
\hline 14 & 250.6846 & 252.4926 & 253.8839 & 757.0611 & 252.3537 \\
\hline 15 & 260.1037 & 261.8931 & 263.2825 & 785.2794 & 261.7598 \\
\hline 16 & 246.4849 & 248.308 & 249.7148 & 744.5078 & 248.1693 \\
\hline 17 & 248.2499 & 249.9993 & 251.3339 & 749.5831 & 249.861 \\
\hline 18 & 257.8956 & 260.1464 & 261.7321 & 779.7741 & 259.9247 \\
\hline
\end{tabular}

Fig. 6 The Results of RGB Total and Mean

Further, it is looked at the analysis and standard deviation of PSNR. These results can be seen in Figure 7. 


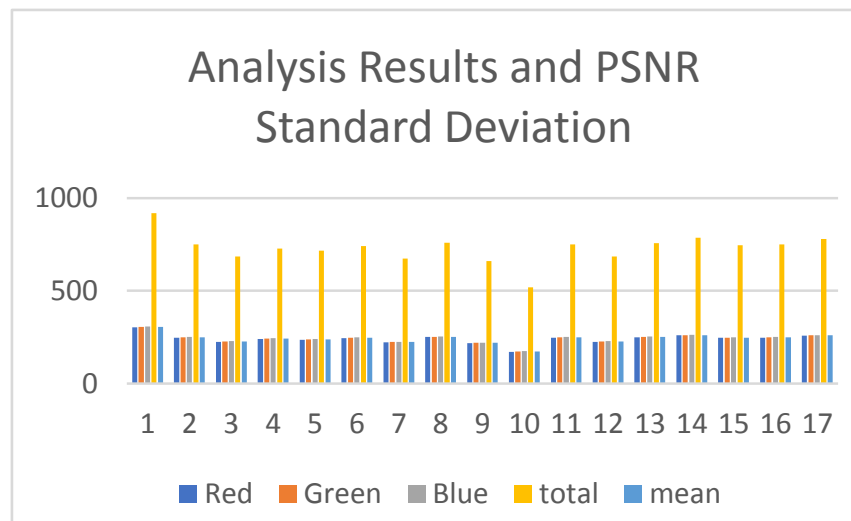

Fig. 7 Analysis Results and PSNR Standard Deviation

The results in Figure 4, is the result of the wavelet transform using the Haar wavelet method, where there are several parameters of measurement error or error in image processing. Two of the most common parameters used are Mean Square Error (MSE) and Peak Signal to Noise Ratio (PSNR).

In Figure 5, it can be seen that the results of the comparison between the R, G and B is not too far away, and at a certain point wavelet density very close distance. Although it is not always correlated with human visual perception, MSE is a good measure to measure the similarity of two images. Suppose we have two images $f$ and $g$ with dimensions as large $\mathrm{Mx} \mathrm{N}$, the greater the value of MSE, the greater the difference between the two pieces of image comparison.

One more parameter measurement error is as good as Pict Signal to Noise Ratio (PSNR). Can be seen in Figure 6 the results of the R, G, B indicates that the overall total of R, G, B when averaged the results are more inclined to color $G$ (green).

From Figure 7, it can be concluded that the total of the R, G, B greatly affect the mean will happen to PSNR is not very far from the range of R, G, B. Another case like MSE, PSNR larger value indicates a closer approximation to the units used PSNR is decibel $(\mathrm{db})$.

\section{CONCLUSION}

From the experiments, can be concluded as follows that the results are obtained the wavelet transform using the Haar wavelet, where there are several parameters of measurement error or error in the processing of the image, but not affected by the dimensions of an image. Two of the most common parameters used are Mean Square Error (MSE) and Peak Signal to Noise Ratio (PSNR). Otherwise, the results of the comparison between the R, G and B is not too far away, and at a certain point wavelet density is very close distance. Although it is not always correlated with human visual perception, MSE is a good measure to measure the similarity of two images. The total of all the R, G, B when averaged the results are more inclined to color $\mathrm{G}$ (green). The total R, G, B greatly affect the mean will happen to PSNR, which is not very far from the range of R, G, B. Another case like MSE,
PSNR larger value indicates a closer approximation to the units used PSNR is decibel (db).

\section{REFERENCES}

[1] Agustini Ketut, "Biometrik Suara dengan Transformasi Wavelet Berbasis Othogonal Daubenchies," Jurnal Teknik Komputer vol. 9, no. 2, pp. 49-56, 2007.

[2] Arisandi Benardinus, Nanik Suciati, dan Arya Yudhi Wijaya, "Pengenalan Motif Batik dengan Rotated Wavelet Filter dan Neural Network, Thesis, 2011.

[3] Sianipar, Rismon H, Sri Muliani WJ, "Kompresi Citra Digital Berbasis Wavelet: Tinjauan PSNR dan Laju Bit," Jurnal Informatika, vol. 4, no. 2, pp. 81-87, 2003.

[4] Maria Alphones, Amlu Anna Joshy, Aneeta Christopher, Anjali B., and Ranjitha Rajan, "Iris Database Compression Using Haar Wavelet Decomposition and Huffman Coding," International Journal of Advanced Research in Computer and Communication Engineering, vol. 5, no. 4, pp. 13-16, April 2016.

[5] Donoho D. L., and Huo Xiaoming, "Uncertainty Principles and Ideal Atomic Decomposition," IEEE Trans. Info. Thry, vol. 47, no. 7, pp. 2845-62, Nov. 2011.

https://doi.org/10.1109/18.959265

[6] Zulfathan, "Metode Pencocokan Citra (Image Matching) Berbasis Koefisien Transformasi Wavelet Diskrit," Thesis at University of Syiah Kuala, Banda Aceh, 2011.

[7] Ingrid Daubechies, "Ten Lectures on Wavelets," CBMS-NSF Regional Conference Series in Applied Mathematics, vol. 61, 1992. https://doi.org/10.1137/1.9781611970104

[8] Rokade Poonam M., Ramesh R. Manza, "Automatic Detection of Hard Exudates in Retinal Images Using Haar Wavelet Transform," International Journal of Application or Innovation in Engineering \& Management, vol. 4, no. 5, pp. 402-410, May 2015.

[9] Waleeda Swaidan, and Amran Hussin, "Haar Wavelet Method for Constrained Nonlinear Optimal Control Problems with Application to Production Inventory Model," Sains Malaysiana, vol. 45, no. 2, pp. 305313, 2016.

[10] Kaur Khushpreet, Sheenam Malhotra, "Image Compression using Haar Wavelet Transform and Discrete Cosine Transform," International Journal of Computer Applications, vol. 125, no. 11, pp. 28-31, Sept. 2015.

[11] Albertus Joko Santoso, Lukito Edi Nugroho, Gede Bayu Suparta, and Risanuri Hidayat, "Compression Ratio and Peak Signal to Noise Ratio in Grayscale Image Compression using Wavelet," International Journal of Computer Science and Technology, vol. 2, no. 2, June 2011.

[12] Singh Y. Shantikumar, B. Phupa Devi, and Kh. Manglem Singh, "Image Compression with Haar Wavelet Transform," International Journal of Computer Applications, vol. 121, no. 9, pp. 15-18, July 2015.

[13] Doretto Gianfranco, Alessandro Chiuso, Ying Nian Wu, and Stefano Soatto, "Dynamic Textures," International Journal of Computer Vision, vol. 51, no. 2, pp. 91-109, 2003.

https://doi.org/10.1023/A:1021669406132

About Author (s):

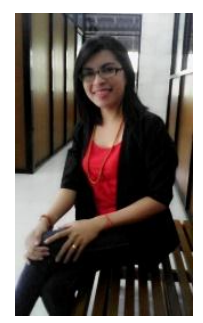

Yustina Rada is a lecturer at Department of Informatics Engineering, University of Wira Wacana, Waingapu, NTT, Sumba, Indonesia. Born in Ngalu, 7th February 1986. She holds M.S. degree in Soft Computing from University of Atma Jaya Yogyakarta. Her research interests are computational, softcomputing, mobile computing, and artificial intelligence.

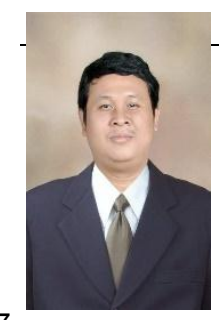

Harrizki Arie Pradana is a lecturer at Department of Informatics Engineering, STMIK Atma Luhur Pangkalpinang, Bangka Indonesia. Born in Pangkalpinang, 13rd April 1986. He holds M.S. degree in Enterprise Information Systems from University of Atma Jaya Yogyakarta. He is a member of APTIKOM and IndoCEISS. His research interests are artificial intelligence, enterprises software and IT. 